\title{
Measures to minimize cross-contamination risks in Advanced Therapy Medicinal Product manufacturing
}

\author{
${\text { Livia } \text { Roseti }^{1}{ }^{*}, \text { Marta Serra }^{1} \text {, and Brunella Grigolo }}^{1,2}$ \\ ${ }^{1}$ RAMSES Laboratory, Research Innovation Technology Department, Rizzoli Orthopaedic Institute, Bologna, Italy \\ ${ }^{2}$ Laboratory of Immunorheumatology and Tissue Regeneration, Rizzoli Orthopaedic Institute, Bologna, Italy \\ *Corresponding author's e-mail address: livia.roseti@ior.it
}

Published online: 19 December 2014 (version 1)

Cite as: Roseti et al., ScienceOpen Research 2015 (DOI: 10.14293/S2199-1006.1.SOR-LIFE.AEJRV9.v1)

Reviewing status: Please note that this article is under continuous review. For the current reviewing status and the latest referee's comments please click here or scan the QR code at the end of this article.

Primary discipline: Life sciences

Keywords: Cross-contamination, Advanced Therapy Medicinal Products, Good Manufacturing Practice, Autologous Chondrocyte Implantation, DNA Profiling

\begin{abstract}
Current European regulations define in vitro expanded cells for clinical purposes as substantially manipulated and include them in the class of Advanced Therapy Medicinal Products to be manufactured in compliance with current Good Manufacturing Practice. These quality requirements are generally thought to be elaborate and costly. However, they ensure three main product characteristics: safety, consistency, and absence of cross-contamination. The term crosscontamination is used to indicate misidentification of one cell line or culture by another. The Good Manufacturing Practice Guidelines suggest some recommendations in order to prevent cross-contaminations and require a demonstration that the implemented actions are effective. Here we report some practical examples useful both to minimize crosscontamination risks in an Advanced Therapy Medicinal Products production process and to evaluate the efficacy of the adopted measures.
\end{abstract}

\section{INTRODUCTION}

Proposed employments for cell-based medicinal products are nowadays quite impressive [1]. Fields of application are musculo-skeletal tissue regeneration, autoimmune disorders, myocardial infarction, gastrointestinal diseases, urogenital system diseases, nervous system disorders, wound healing, plastic surgery, organ transplantation, graft versus host disease, and diabetes. Current European cell therapy laws have recently classified cell manipulation types, according to potentially associated risks. Induction to proliferation, nonhomologous use (not intended to be utilized for the same essential functions in the recipient as in the donor), and an eventual association with scaffolds or medical devices have been termed "substantial manipulations". This has defined the class of Advanced Therapy Medicinal Products (ATMPs) [2] that have to be mandatorily produced in proper facilities and in accordance with current Good Manufacturing Practice (GMP) Guidelines [3]. Such quality requirements are generally thought to be elaborate and costly. However, they ensure three main ATMP characteristics: safety, consistency, and absence of cross-contamination. The term cross-contamination is used to indicate misidentification of one cell line or culture by another [4]. This is a major problem to consider when producing ATMPs. For example, in autologous cell therapy, if accidental cross-contamination between two cultures from two different patients occurs during production in a GMP facility, the relative mixed-up ATMPs would carry potential implications for donor engraftment. To this end, the GMP Guidelines suggest some recommendations in order to prevent cross-contamination and require a demonstration that the implemented actions are effective.

Here we report some practical examples useful both to minimize cross-contamination risks in an ATMP production process and to evaluate the efficacy of the adopted measures.

\section{TECHNICAL, ORGANIZATIONAL, AND BEHAVIORAL MEASURES}

Actions to be implemented to minimize cross-contamination risks during ATMP production are described in general in Chapter 5 of Volume 4 - EU Guidelines for Good Manufacturing Practices for Medicinal Products for Human and Veterinary use, Current Edition. In the practical applications they may be adapted or implemented, depending on cell type, manipulation process, or therapy.

\section{Environment}

The GMP facility should be designed and subsequently built in order to reduce to minimum the recirculation or re-entry of untreated or insufficiently treated air. Pressure differential and airlocks should ensure flow reversal containment and 
prevention, without creating turbulence. Appropriate air extraction should be provided in each clean room, depending on classification (A, B, C, and D areas) [5]. Pass boxes for raw materials handling should be constructed. Dedicated and selfcontained zones should be available for particular products such as highly sensitizing materials or biological preparations intended for injection. GMP facility-specific parameters like air filtration and ventilation, temperature, relative humidity, differential pressure, number of air particles, and bacterial colony forming units should be standardized and continuously monitored.

\section{Personnel}

Only trained and equipped operators can enter the GMP facility. Personnel should wear disposable sterile garments that both ensure protection against hazardous dry particles, aerosols, liquids, and small size particles and represent a physical barrier for cross-contamination (Figure 1).

\section{Cleaning procedures}

Ineffective cleaning of environment and equipment is a common source of cross-contamination. Therefore, decontamination and cleaning procedures should be validated in order to verify their effectiveness (tests for product residues presence) [6]. A rigorous cleaning program should be applied for the routine activities by trained dedicated personnel. Validated extraordinary procedures should be followed after accidental cross-contamination occurs.

\section{Production process}

Different cell-based products should not be manipulated simultaneously. Production in segregated areas is recommended. When this is not possible, due to the structure small dimensions, a production by campaign (separation in time) followed by appropriate and validated cleaning procedures is suggested. Closed, automated systems of production should be preferred. When culture operations are manual more rigorous procedure should be developed and followed.

\section{Raw materials}

Sterile and disposable plastic wares must be utilized. Cell culture media sterility should be certified to be negative for aerobic, anaerobic bacteria, fungi, and endotoxins. Animal origin reagents need to be tested also for mycoplasma and viruses presence. If fetal bovine serum is used, its origin from countries with negligible Bovine Spongiform Encephalopathy (BSE) and Transmissible Spongiform Encephalopathy (TSE) risks should be certified [7]. In general, the material's full batch documentation/certification should be carefully evaluated to attest fulfillment with specific current regulations.

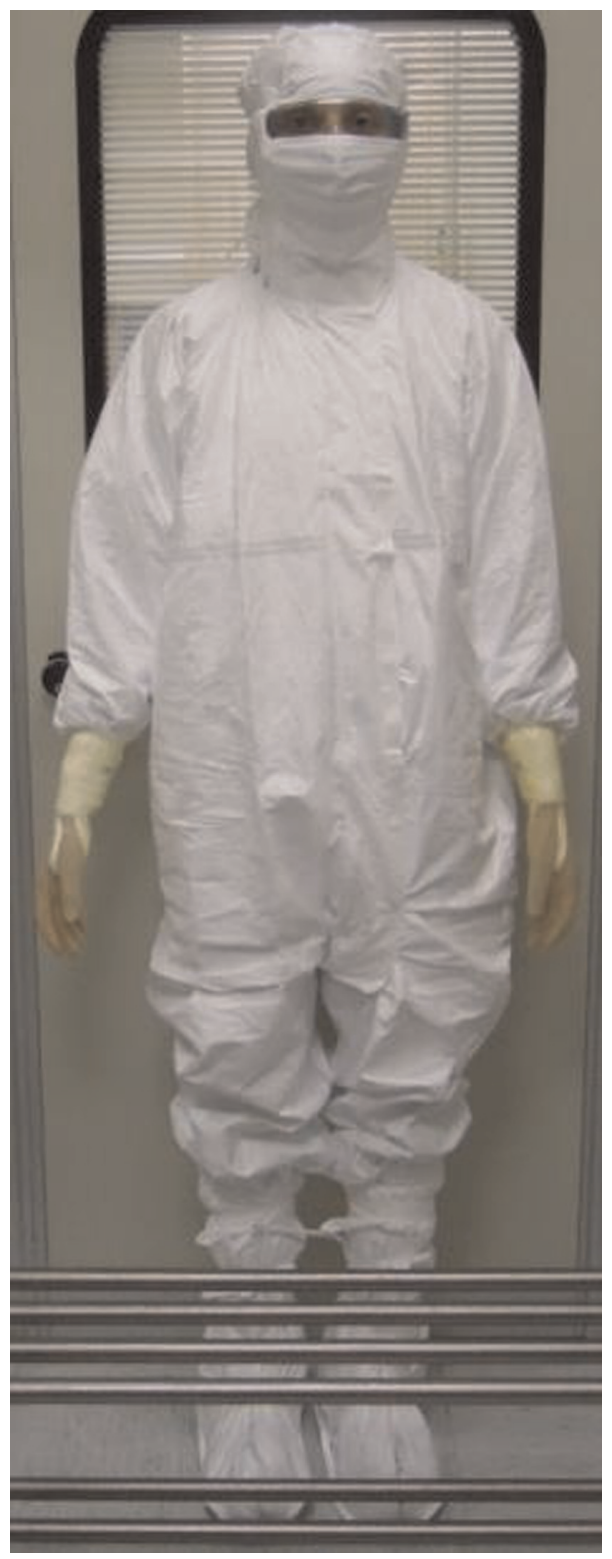

Figure 1. Personnel should wear disposable sterile garments that both ensure protection against particles, aerosols, and liquids, and represent a barrier to cross-contamination.

\section{Donor screening}

Donors should be evaluated according to current legislation [8]. In particular, blood samples should be harvested to evaluate the presence of transmissible pathologies by means of HIV-1/HIV-2 Antibody, Hepatitis B Surface Antigen, Hepatitis B Core Antibody, Hepatitis C Virus Antibody, and syphilis screening. In addition, nucleic acid techniques (NAT) for HBV DNA, HCV RNA, HIV DNA detection should performed to reveal virus presence also during the window period. Positive donors' cultures should be manipulated in segregated areas to avoid cross-contamination risks. For instance, it has been 
demonstrated that Human Bone Marrow Mesenchymal Stem Cells in culture [9] are able to fully support HBV infection, replication, expression, and secretion. If the GMP facility does not display the possibility to treat infected cells in segregated areas, positive patients should be previously excluded from the treatment.

\section{HOW TO PROVE THE ABSENCE OF CROSS- CONTAMINATION IN AN ATMP}

In general, GMP compliance protects from cross-contamination risks. However, it is required to demonstrate that the adopted measures are effective. An option that has been already proved to be useful for this aim is the DNA profiling technique $[10,11]$. This is a highly sensitive and specific method routineously used in forensic caseworks for human identification [12] and applied to reveal and monitor microchimerism after stem cell transplantation [13]. DNA profiling is the determination of unique genetic characteristics that make an individual distinguishable from of all other humans, including closely related ones. In particular, this technique allows the identification of short, highly specific, tandemrepeated (hypervariable) genomic sequences (short tandem repeats, STRs), that do not contribute to gene function. Based on a specific statistical approach, the random match probability is equated with the probability that a match would occur by chance. The random match probability is the unlikely coincidence that an unrelated person would by chance have the same DNA profile and can be determined by calculating the frequency of the observed profile in a reference population database [14].

As a practical example, we report the use of DNA profiling in the context of the Autologous Chondrocyte Implantation (ACI) technique [10], a worldwide applied cell therapy. Regenerative procedures, including ACI, can be used as second-line measures to repair chondral or osteochondral defects. ACI therapeutical approach was first used to treat full-thickness chondral defects of the knee and later applied to the ankle [15]. In the original procedure, small grafts of normal cartilage removed from non-weight bearing areas of the knee were treated in a GMP facility. Chondrocytes were isolated, expanded in culture for several weeks, and then retrieved as a suspension for re-implantation. Recent experimental and clinical research has been directed toward the development of procedures using suitable scaffolds which act as carriers for the implantable cells, maintaining at the meantime phenotype stability [16]. In this case, DNA profiling need to be performed both on blood samples of patients undergoing arthroscopy for ACI and on final ATMPs (cells harvested at the end of the culture period). Specific and validated kits analyzing 15 tetranucleotide STRs plus amelogenin locus for sex determination (internal control) can be utilized. If the results evidentiate that the unique DNA profile of each individual blood sample is maintained unaltered in its corresponding ATMP, this is an evidence of cross-contamination absence between different chondrocyte cultures. In fact, the genetic profile of each patient detected in blood sample controls before manipulation is unaltered in the cells until the end of the production process [10].

\section{CONCLUSION}

When producing ATMPs it is mandatory to implement and give evidence of all the possible measures to apply to prevent/minimize cross-contamination risks.

If accidental cross-contamination occurs or is revealed by evidence tests (i.e. DNA profiling), extraordinary cleaning procedures should be applied and an investigation performed in order to find the causes, if any. Preventive actions and monitoring should be implemented in order to avoid or reduce this risk in the future. In general, contaminated ATMPs should be eliminated.

\section{ACKNOWLEDGMENTS}

The authors wish to thank Dr Alessandra Bassi for her helpful advice.

\section{REFERENCES}

[1] Mason C, Manzotti E. Regenerative medicine cell therapies: numbers of units manufactured and patients treated between 1988 and 2010. Regen Med. 2010;5(3):307-13. doi:10.2217/ rme.10.37

[2] Regulation (EC) No 1394/2007 of the European Parliament and of the Council on advanced therapy medicinal products and amending Directive 2001/83/EC and Regulation (EC) No $726 / 2004$.

[3] European Commission, The Rules Governing Medicinal Products in the European Union. Volume 4 - EU guidelines to good manufacturing practice medicinal products for human and veterinary use.

[4] Schaffer WI, Tissue Culture Association Terminology Committee. Terminology associated with cell, tissue, and organ culture, molecular biology, and molecular genetics. In vitro Cell Dev Biol. 1990; 26(1):97-101. doi:10.1007/BF02624162

[5] European Commission, The Rules Governing Medicinal Products in the European Union. Volume 4 - EU guidelines to good manufacturing practice medicinal products for human and veterinary use, annex 1 - manufacture of sterile medicinal products, current edition.

[6] European Commission, The Rules Governing Medicinal Products in the European Union. Volume 4 - EU guidelines to good manufacturing practice medicinal products for human and veterinary use, annex 15 - qualification and validation, current edition.

[7] Committee for Medicinal Products for Human Use (CHMP). Guideline on the use of bovine serum in the manufacture of human biological medicinal products, 30 May 2013 (EMA/CHMP/ BWP/457920/2012 rev 1).

[8] Directive 2004/23/EC of the European Parliament and of the Council of 31 March 2004 on setting standards of quality and safety for the donation, procurement, testing, processing, preservation, storage and distribution of human tissues and cells.

[9] Ma R, Xing Q, Shao L, Wang D, Hao Q, Li X, Sai L, Ma L. Hepatitis $B$ virus infection and replication in human bone marrow mesenchymal stem cells. Virol J. 2011;8(1):486. doi:10.1002/ hep.1840180406

[10] Roseti L, Bassi A, Fornasari PM, Serra M, Canella F, Maso A, Dallari D, Bini C, Pelotti S. A novel DNA profiling application for the monitoring of cross-contamination in autologous 
chondrocyte implantation. Eur Rev Med Pharmacol Sci. 2013; 17(6):820-33.

[11] Roseti L, Serra M, Canella F, Munno C, Tosi A, Zuntini M, Pandolfi M, Sangiorgi L, Biso P, Pittalis MC, Bini C, Pelotti S, Gasbarrini A, Boriani L, Bassi A, Grigolo B. In vitro gene and chromosome characterization of expanded bone marrow mesenchymal stem cells for musculo-skeletal applications. Eur Rev Med Pharmacol Sci. 2014; 18(23):3702-11.

[12] Lins AM, Micka KA, Sprecher CJ, Taylor JA, Bacher JW, Rabbach DR, Bever RA, Creacy SD, Schumm JW. Development and population study of an eight-locus short tandem repeat (STR) multiplex system. J Forensic Sci. 1998; 43:1168-80.

[13] Kristt D, Israeli M, Narinski R, Or H, Yaniv I, Stein J, Klein T. Hematopoietic chimerism monitoring based on STRs: quantitative platform performance on sequential samples. J Biomol Tech. 2005; 16(4):380-91.

[14] Foreman LA, Evett IW. Statistical analyses to support forensic interpretation for a new ten-locus STR profiling system. Int J Legal Med. 2001;114(3):147-55. doi:10.1007/s004140000138

[15] Brittberg M, Lindahl A, Nilsson A, Ohlsson C, Isaksson O, Peterson L. Treatment of deep cartilage defects in the knee with autologous chondrocyte transplantation. N Engl J Med. 1994; 331(14):889-895. doi:10.1056/NEJM199410063311401

[16] Iwasa J, Engebretsen L, Shima Y, Ochi M. Clinical application of scaffolds for cartilage tissue engineering. Knee Surg Sports Traumatol Arthrosc. 2009; 17(6):561-77. doi:10.1007/s00167008-0663-2

\section{COMPETING INTERESTS}

The authors declare no competing interests.

\section{PUBLISHING NOTES}

(C) 2014 Livia Roseti et al. This work has been published open access under Creative Commons Attribution License CC BY 4.0, which permits unrestricted use, distribution, and reproduction in any medium, provided the original work is properly cited. Conditions, terms of use and publishing policy can be found at www.scienceopen.com.

Please note that this article may not have been peer reviewed yet and is under continuous post-publication peer review. For the current reviewing status please click here or scan the QR code on the right.

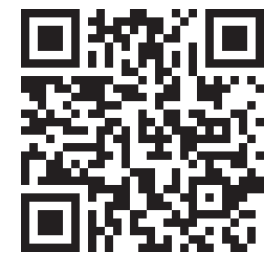

\section{ScienCeOPEN.com}

